Artigo de ReVISÃo

REVIEW ARTICLE

\title{
Imunidade na Gestação Normal e na Paciente com Lúpus Eritematoso Sistêmico (LES) ${ }^{(*)}$
}

\section{Immunity in the Normal Presnancy and in the Patient with Systemic Lupus Erythematosus (SLE)}

\author{
Alessandra Cardoso Pereira ${ }^{(1)}$, Nilson Ramires de Jesús ${ }^{(2)}$, Lais Verderame Lage ${ }^{(3)}$, Roger Abramino Levy ${ }^{(4)}$
}

\section{RESUMO}

A gravidez é uma condição fisiológica na qual ocorrem várias mudanças imunoendócrinas com a finalidade de facilitar a imunossupressão e a tolerância aos antígenos paternos e fetais. $\mathrm{Na}$ gravidez humana normal existe uma relativa supressão de citocinas tipo Th1 na resposta dos linfócitos, levando a uma prevalência na resposta do tipo Th2. No LES, onde prevalece a resposta imune do tipo Th2, a gravidez pode estar relacionada com a ativação da doença. Este artigo é uma revisão dessas alterações relacionadas com a resposta imune durante a gestação normal e na da paciente com LES.

Palavras-chave: gravidez, lúpus eritematoso sistêmico, resposta Th2.

O período gestacional representa um modelo único na natureza. Compreende um estado imunológico altamente complexo, com freqüente exacerbação de enfermidades ou alterações pré-existentes. Do ponto de vista imunológico tem sido um grande desafio tentar entender quais os mecanismos envolvidos na não rejeição da placenta pelo sistema imunológico materno, já que a mesma, por ser de origem embriônica, contém material genético tanto materno como paterno. Assim sendo, apresenta potencial para expressar antígenos paternos, que são geneticamente estranhos à mãe e, portanto, poderiam incitar as mesmas reações de intolerância imunológica observadas nos tecidos

\begin{abstract}
Pregnancy is a physiologic condition where several immunoendocrine changes take place with the aim of facilitating immunosuppression and tolerance towards paternal and fetal antigens. In normal human pregnancy there is a relative suppression of Th1 type cytokines in response to lymphocytes, leading to a Th2 type response. In SLE, where a Th2 type response prevails, pregnancy may exacerbate the disease. This article reviews the alterations related to the immune response during normal pregnancy and in SLE patients.
\end{abstract}

Keywords: pregnancy, systemic lupus erythematosus, Th2 response.

enxertados $^{(1)}$. Essa importante observação foi inicialmente referida pelo imunologista de transplantes Peter Medawar, em 1953 ${ }^{(1)}$. Desde então, mesmo após cinco décadas de intensas pesquisas sobre a imunologia da reprodução, ainda não se conhece perfeitamente o mecanismo de adaptação imunológica presente na gestação, que permite o sucesso do enxerto placentário.

O desenvolvimento da gestação depende do estabelecimento de um sistema vascular eficiente, que supra oxigênio e nutrientes de forma adequada ao produto conceptual. Para o estabelecimento desse "novo" sistema vascular (placentação), o trofoblasto infiltra-se pela parede endome-

* Trabalho realizado nas Disciplinas de Reumatologia e de Obstetrícia do Hospital Universitário Pedro Ernesto da Universidade do Estado do Rio de Janeiro (HUPE/UERJ). Rio de Janeiro, RJ, Brasil. Recebido em 05/03/2005. Aprovado, após revisão, em 28/05/2005.

1. Professora assistente da Disciplina de Reumatologia da Faculdade de Medicina da Universidade do Grande Rio (Unigranrio), Rio de Janeiro, RJ, Brasil e mestranda de Ciências Médicas do PGCM da UERJ.

2. Professor assistente da Disciplina de Obstetrícia do HUPE/UERJ.

3. Professora colaboradora da Disciplina de Reumatologia da Faculdade de Medicina da Universidade de São Paulo (FMUSP), São Paulo, SP, Brasil e Assistentedoutora do Serviço de Reumatologia do Hospital das Clínicas da FMUSP.

4. Professor adjunto da Disciplina de Reumatologia do HUPE/UERJ.

Endereço para correspondência: : Dra. Alessandra Cardoso Pereira. Rua Barão de Mesquita, 931 - ap. 101, Tijuca, CEP 20540-002. Rio de Janeiro, RJ, Brasil. Tel. 55 (21) 9948-0820; fax 55 (21) 2567-7530; e-mail: lelesandy@terra.com.br 
trial, até alcançar as artérias espirais uterinas. A seguir, o citotrofoblasto recobre a decídua com destruição da camada muscular arterial, transformando-a em vasos de baixa resistência. Durante esse processo, o citotrofoblasto substitui seu fenotipo originariamente epitelial, pelo fenotipo endotelial, recobrindo a camada endovascular das artérias espirais maternas ${ }^{(2)}$. A placentação permite que a artéria uterina se expanda na medida em que a gestação progride, evitando a restrição de fluxo sanguíneo à unidade fetoplacentária. Por outro lado, isso faz com que produtos de genes fetais, assim como antígenos específicos de diferenciação sejam expostos a células maternas imuno-competentes presentes no local ${ }^{(3)}$.

O sistema imune inato está ativo na gravidez, e em razão da relativa supressão da imunidade adaptativa, pode assumir um importante papel na defesa imune materna ${ }^{(4)}$. Além disso, o sistema imune inato tem um papel de influência dominante sobre a reprodução. Análises dos tipos celulares que estão presentes no local da implantação do óvulo mostram que as células $\mathrm{T}$ e $\mathrm{B}$, típicas do sistema imune adaptativo, são raras, enquanto o predomínio populacional consiste de células $\mathrm{NK}^{(5)}$.

Nas fases iniciais de implantação ovular, durante o reconhecimento da presença do "semi-enxerto", i.e., o trofoblasto, estabelece-se uma resposta imunoalogênica de natureza inflamatória, similar à fase inicial de rejeição de enxerto. O trofoblasto forma uma coluna de células mononucleadas que invade a decídua uterina e seus vasos sanguíneos para prover ancoragem e suprimento sanguíneo ao feto em desenvolvimento. Portanto, a apresentação de antígenos pelo trofoblasto na interface materno-fetal parece ser um componente importante de resposta imunologia materna durante a gestação.

Duas teorias principais tentam explicar o fenômeno gestacional. Uma que enfatiza o papel das reações imunossupressoras para a proteção do feto. Por outro lado, a teoria do "imunetropismo" defende a importância da resposta imunológica materna aos antígenos paternos presentes no concepto.

O endométrio humano é um tecido dinâmico, que ao se preparar para a implantação do óvulo, passa por ciclos bem definidos de proliferação, diferenciação e degradação em resposta à prevalência hormonal do meio. A população leucocitária dentro do estroma endometrial também varia durante o ciclo menstrual e ao longo da gravidez. Leucócitos endometriais incluem células T e B, mastócitos, macrófagos e neutrófilos, além de células natural killer uterinas $(\mathrm{uNK})^{(6)}$. Os principais tipos de células imunes no endo- métrio secretório são as células T, células uNK e os macrófagos. As células T compreendem aproximadamente 45\% dos leucócitos na fase proliferativa do endométrio, e esse número permanece constante em toda a fase secretória e na gestação. Contudo, seu número aparentemente diminui em decorrência do grande aumento das células uNK durante a fase secretória e no início da gravidez ${ }^{(7)}$.

No primeiro trimestre de gestação, 70\% a 80\% dos linfócitos presentes na mucosa uterina são linfócitos granulares grandes, que representam menos de $1 \%$ da população dos linfócitos do sangue periférico. Embora esses linfócitos sejam fenotipicamente e morfologicamente classificados como células NK, são menos citotóxicos que as células NK do sangue periférico $(\mathrm{pNK})^{(8)}$. Os leucócitos granulados deciduais (LGDs) têm uma composição incomum: aproximadamente $70 \%$ são células uNK, células NK especializadas, $\left[\left(\mathrm{CD} 56^{\text {bright }} \text { e } \mathrm{CD} 16^{-}\right)^{(5)}\right.$ e $\left.\left(\mathrm{CD}^{-}\right)^{(7)}\right]$, e o restante inclui proporções iguais de monócitos (aproximadamente 15\%) e células T (aproximadamente 15\%) ${ }^{(9)}$. As uNK constituem $70 \%$ dos linfócitos residentes na decíduas da grávida humana, em contraste com as pNK, que são menos de 15\% dos linfócitos circulantes ${ }^{(10)}$. Ademais, o fenótipo das pNK $\left(\mathrm{CD} 56^{\mathrm{dim}}, \mathrm{CD} 16^{\text {bright }}\right.$ e $\left.\mathrm{CD}^{-}\right)$é diferente das $\mathrm{uNK}^{(6)}$. Além disso, esses linfócitos uterinos proliferam, ativam-se na mucosa e são uma importante fonte de citocinas como o fator inibidor de leucemia (leukemia inhibitory factor - LIF) e fator estimulador de colônia de macrófagos (macrophagecolony stimulating factor $-\mathrm{M}-\mathrm{CSF})^{(8)}$, fator de necrose tumoral (TNF), interferon- $\gamma$ (INF- $\gamma$ ) e interleucina -10 $(\text { IL-10) })^{(7)}$, as quais têm importante papel na implantação e crescimento da placenta ${ }^{(8)}$.

Vários genes imunes foram considerados como reguladores do período de implantação, incluindo o fator declínio de aceleração (DAF - decay accelerating factor), indoleamina 2,3 dioxigenase (IDO), IL-15, IL-15R $\alpha$, fator regulador de interferon-1 (IRF-1), linfotoxina, transcriptase 2 associado a NK (NKAT2) e NKG5. Esses genes cobrem uma extensa gama de funções, incluindo a promoção da proliferação das células uNK, quimiotaxia, inibição da atividade citolítica das células NK, inibição do crescimento celular (células T e patógenos) e inibição da via clássica do complemento. Estes genes representam uma diversidade de funções que provavelmente têm como objetivo a preparação do endométrio para implantação com especial ênfase em adaptar o sistema materno para acomodar o feto, "estranho" imunológico ${ }^{(7)}$.

O sucesso de uma gravidez depende do estabelecimento e manutenção de um sistema vascular útero-placentário 
eficiente. Durante a gestação, como em qualquer outro processo imunoinflamatório, a unidade útero-placentária inicia e modula uma interação harmônica entre o endotélio vascular materno, as células imunocompetentes presentes localmente, os determinantes antigênicos presentes na superfície do trofoblasto, ativamente regulando o processo de adesão, ativação e migração celular, via modificações na rede de citocinas locais.

O status antigênico do embrião no período pré-implantação não é bem definido e, portanto, não há reação materna de reconhecimento imunológico neste estágio ${ }^{(11)}$.

Os mecanismos pelos quais os hemialogênicos placentários humanos evitam a rejeição pelo sistema imune materno ainda se encontram em investigação. De acordo com o papel placentário central na manutenção da gravidez, evidências sugerem vários níveis de regulação. Existem vários exemplos de fatores solúveis produzidos pelo trofoblasto fetal com potencial de atividade de modificar o sistema imune materno.

Ambas as invasões do citotrofoblasto e do LGDs têm propriedades imunológicas incomuns. Por exemplo, as células fetais expressam o MHC não clássico classe Ib moléculas HLA-G e HLA-E, em vez de as clássicas moléculas HLA-A e HLA-B. A expressão do HLA-C pelo citotrofoblasto também foi descrita ${ }^{(9)}$. Embora a função precisa das células uNK ainda seja desconhecida, a proximidade delas com a invaginação trofoblástica, a qual seletivamente expressa HLA-C, HLA-E, HLA-G e moléculas CD 1d, tem conduzido à proposta que estes antígenos de MHC no trofoblasto interagem com receptores das células $\mathrm{NK}^{(10)}$. Ainda está obscuro, porém, quais são os receptores das células uNK que interagem com as moléculas HLA expressas no trofoblasto, e se tais interações inibem a lise da célula NK ou levam a produção de citocinas que favorecem o desenvolvimento normal da placenta e a manutenção da gravidez ${ }^{(10)}$. Além disso, a interação entre os receptores de células NK e seus ligantes tem sido estudada principalmente nas células pNK, mas a relação entre células uNK e células pNK continua desconhecida ${ }^{(10)}$. As conseqüências funcionais da interação entre citotrofoblasto e LGDs não estão bem esclarecidas, mas experiências com camundongos sugerem que as células imunes promovem a invasão endovascular e o desenvolvimento placentário ${ }^{(9)}$.

Várias observações sugerem que as células uNK têm um importante papel na reprodução: elas são reguladas hormonalmente, aumentando em número durante a fase lútea do ciclo menstrual quando ocorre a implantação; estão presentes no início da gestação na ocasião em que as células trofoblásticas invadem as arteríolas espiraladas. São particularmente abundantes em torno da infiltração fetal derivada de células trofoblásticas extravilosas. Células CD56 ${ }^{+}$proliferam ativamente na decídua durante a fase lútea. Nessas fases outros elementos da mucosa (glândulas epiteliais e células do estroma) cessam a proliferação e iniciam a diferenciação, exceto pelas células endoteliais, as quais continuam a proliferar nessa ocasião. Contudo, o estímulo para a proliferação in vivo da célula NK ainda é desconhecido ${ }^{(12)}$. A associação entre a morte das células uNK e a queda dos níveis de progesterona, assim como a natureza cíclica desse aparecimento, parecem sugerir regulação hormonal dessas células. Até o momento, porém, não foi possível localizar os receptores de estrogênio ou de progesterona dessas células. Acredita-se que estrogênio e progesterona possam exercer seus efeitos sobre as células uNK indiretamente através de citocinas, como IL-15, e da prolactina (PRL) ou de outros fatores solúveis ${ }^{(3)}$. As células uNK expressam receptores para glicocorticóide e o receptor $\beta$ de estrogênio, sugerindo que essas respondem a estes hormônios ${ }^{(13)}$.

A IL-2 é conhecida por sua importante função de proliferação e ativação das uNK in vitro; apesar de os receptores de expressão com alta afinidade para IL-2 compostos por cadeia $\alpha, \beta$ e o comum $\gamma$ nas uNK serem de difícil detecção no endométrio e decíduas não patológicos. Outro candidato, o fator de célula tronco (stem cells), não induz a proliferação das células uNK na ausência de IL-2; assim, o mecanismo que controla a proliferação e sobrevivência de uNK não é completamente conhecido(8).

A IL-15 tem um papel crucial no sistema imune inato: é uma citocina essencial para o desenvolvimento e manutenção da célula $\mathrm{NK}^{(14)}$; é produzida na mucosa uterina, e pode estar relacionada com a citotoxicidade e proliferação das células uNK; parece promover um papel regulador das células uNK in vivo ${ }^{(12)}$. Sua estrutura molecular e função biológica são semelhantes a da IL-2, mas sua seqüência e localização tecidual diferem das da IL-2. A IL-15 pode proliferar uma população inteira de linfócitos, incluindo células NK. Os receptores (R) de IL-15 se assemelham aos receptores da IL-2, pois são compostos de três subunidades $^{(8)}$ : o receptor de cadeia $\gamma$, que é compartilhado pela IL-15 e pela IL-2; a IL-15Rß; e a IL-15R $\alpha^{(14)}$. Tais semelhanças entre a IL-2 e a IL-15 sugerem que a IL-15 possa ser um potente ativador das células $\mathrm{uNK}^{(8)}$. Estudos sugerem que os macrófagos sejam uma importante fonte de IL-15 no útero ${ }^{(12)}$.

Essas moléculas incluem citocinas e fatores de crescimento, como IL-10, fator transformador de crescimento 
B 1 (TGF-B1 - transforming growth factor-B1) e proteína inflamatória de monócito (MIP), que diminuem a regulação da resposta imune em outros locais ${ }^{(9)}$. A placenta também adota estratégias para regular o sistema imune na produção de um nível metabólico da enzima indoleamina 2,3-dioxigenase, as quais catabolizam o triptofano necessário para ativar as células $\mathrm{T}^{(9)}$. Finalmente, vários mecanismos insólitos de regulação imune são usados na interface materno fetal, incluindo aumento do nível local de progesterona e expressão da molécula de MHC não clássica classe I denominada HLA-G, a qual também tem uma forma solúvel. Todos esses produtos diminuem a regulação da resposta imune, particularmente a atividade das células T e NK, os mediadores primários dos tecidos de rejeição halogênicos ${ }^{(9)}$.

O denominado "paradigma de Th1/Th2" parece explicar uma pergunta desafiadora, por que o feto não é rejeitado pelo sistema imune materno apesar da presença dos antígenos paternos em contato com as células imunocompetentes maternas ${ }^{(15)}$.

$\mathrm{Na}$ interface materno fetal as citocinas do tipo Th2, que são secretadas não somente pelas células imunocompetentes mas também por células da decídua e da placenta, parecem ser as responsáveis pelo sucesso na manutenção da gravide $z^{(15)}$.

Isso foi embasado na observação de que, IL-4, IL-10 e IL-3 foram detectadas em níveis aumentados durante a gravidez normal em humanos e animais. Além disso, a injeção de citocinas Th2 poderia prevenir o aborto em camundongos. Ao contrário, a produção excessiva de citocinas Th1 foi associada com perda gestacional, especialmente citocinas tal como IFN- $\gamma$, TNF, IL-1 e IL-2. Assim, a produção aumentada de citocinas Th2, oposta pela produção diminuída de citocinas Th1, parecia ser a razão perfeita para explicar a sobrevivência do feto no útero materno. Entretanto, estudos recentes não mostraram diferenças na secreção de INF- $\gamma$ pelos linfócitos de pacientes que apresentaram aborto espontâneo comparado com pacientes com gestação normal ${ }^{(15)}$. Zenclussen et al. encontraram níveis de IL-12 e citocina indutora Th1 aumentados no primeiro trimestre de gestação normal; isso parece ser indicativo de que citocinas inflamatórias podem não ser prejudiciais como tem sido sugerido pelo paradigma Th1/Th2. A presença de IFN- $\gamma$ é necessária para a manutenção da decídua em humanos. IFN- $\gamma$ é secretado pelas uNK, é responsável pela remodelação dos vasos da decídua induzidos pela gravidez, e necessário para celularidade e integridade decidual. Além disso, receptores para IFN- $\gamma$ estão expressos em várias células que estão presentes nos locais de implantação ${ }^{(15)}$. Um estudo realizado por Parr et al. verificou que o TNF liberado pelas
uNK no começo da gestação tem um papel fundamental na gravidez em camundongos, estando relacionados com: 1 - proteção da mãe contra as células anormais fetais; 2 restrição do trofoblasto à invasão da decídua materna; 3 facilitação da remodelação do tecido requerido para acomodação do embrião; 4 - proteção contra transmissão de microorganismos da mãe para o feto ${ }^{(16)}$.

A placenta e as membranas fetais contêm e expressam mRNA para TNF e TGF-B1, além desses tecidos serem uma fonte importante dessas citocinas. O TNF e o TGF-B1 têm vários papéis importantes na gestação a termo. Estão envolvidos na regulação local das prostaglandinas produzidas pelas membranas fetais, onde apresentam efeitos contrários. O TNF estimula fortemente a liberação de prostaglandina E2 (PGE2) por esses tecidos. Por outro lado, o TGF-B1 inibe as citocinas estimuladoras da PGE2. Esses efeitos, contudo, já foram questionados em outros estudos. Os níveis de TGF-B1 no plasma materno parecem aumentar progressivamente até o final da gestação normal e também aumentam em mulheres com abortos recorrentes. Essas citocinas TGF-B1 e TNF também estão envolvidas no crescimento e diferenciação do trofoblasto, na produção de outras citocinas como IL-6 e IL-8, na expressão de moléculas de adesão, e na biogênese das metalaproteinases da matriz. Essas ações podem estar envolvidas no processo de trabalho de parto. A oxitocina, progesterona e hidrocortisona podem influenciar significativamente na expressão do mRNA e na liberação tecidual de TGF-B1 e TNF pelas membranas fetais na gestação a termo ${ }^{(17)}$.

$\mathrm{Na}$ gravidez humana normal a relativa supressão de citocinas tipo Th1 na resposta dos linfócitos, produzindo uma propensão Th2, é acompanhada por uma intensificação moderada ou igual da resposta dos monócitos a favor das citocinas pró-inflamatórias ${ }^{(18)}$.

Embora alguns papéis fisiológicos das citocinas próinflamatórias da interface materno fetal sejam descritas com relação ao crescimento da placenta e decídua, a produção excessiva ou aberrante de citocinas pró-inflamatórias, tal como a IL-1B, TNF, e IFN- $\gamma$ na interface materno fetal, é prejudicial para a gravidez. A IL-10 é uma citocina importante porque suprime a produção de citocinas pró-inflamatórias por outras células. Numerosos estudos têm documentado a produção da IL-10 na interface materno fetal. A gravidez não é dependente da produção de IL-10, o que sugere que outros imunomoduladores também podem contribuir para a sobrevivência do enxerto fetal ${ }^{(19)}$.

O aumento fisiológico das concentrações de cortisol, progesterona, estradiol e testosterona, observado durante 
o terceiro trimestre, estão envolvidos na polarização das citocinas $\mathrm{Th} 2^{(20)}$. Durante a gravidez, a produção de citocinas pelos linfócitos é principalmente do tipo Th2; os linfócitos periféricos liberam menos IFN- $\gamma$ e IL-2 e mais IL-4 e IL-10, particularmente no terceiro trimestre de gestação ${ }^{(21,22)}$. Existe uma complexa interação hormonal, que acarreta a supressão de IL-12 e TNF (resposta Th1), com aumento da resposta $\mathrm{Th} 2^{(23)}$.

$\mathrm{O}$ imunomodulador melhor estudado da interface materno fetal é a progesterona $\left(\mathrm{P}_{4}\right)$, que tem um papel evidente na sobrevivência do enxerto fetal. Numerosas experiências têm demonstrado que a progesterona bloqueia o estimulador da proliferação mitogênica linfocitária, melhora o tempo de sobrevivência do enxerto, modula a produção de anticorpos, diminui a quebra oxidativa dos monócitos, reduz a produção de citocinas pró-inflamatórias pelos macrófagos na resposta a produtos bacterianos, e altera a secreção de citocinas dos clones das células $\mathrm{T}$ para favorecer a produção de IL-10 ${ }^{(19)}$. O mecanismo pelo qual a progesterona exerce ações imunomoduladoras nos tecidos reprodutivos ainda não está claro, mas envolve ações diretas e indiretas sobre as células imunes ${ }^{(19)}$. Proteínas com atividade imunossupressora que são produzidas sob influência de progesterona têm sido descritas no endométrio de ruminantes e leucócitos humanos. Tais proteínas podem alterar a proliferação, ativação e função efetora das células imunes na interface materno fetal e podem prover uma alternativa, com um mecanismo indireto para imunossupressão mediado por progesterona ${ }^{(19)}$.

\section{HORMÔNIOS E GESTAÇÃO NO LES}

A gravidez nos mamíferos é caracterizada por um aumento de progesterona, estrogênio e prolactina, além de vários hormônios placentários ${ }^{(24)}$. A gravidez é um estado de predomínio de estrogênio que está associado com perfil de citocinas Th2, essencial para a tolerância materna ao feto e manutenção da gravidez. No LES, onde prevalece a imunidade Th2, a gravidez está relacionada com a ativação da doença ${ }^{(25)}$. Estrogênio promove a maturação das células B e formação de anticorpos, além de evitar a apoptose dos macrófagos na sinóvia, e reduzir a apoptose das células do sangue, mas não das células T. O aumento do estrogênio e diminuição do androgênio aumenta o desenvolvimento e gravidade do lúpus em ratos, enquanto que a supressão do estrogênio retarda o processo da doença. Pacientes com LES de ambos os sexos têm aumento da hidroxilação de estrógenos em 16- $\alpha$-hidroxiestrona e estriol, metabólitos feminilizantes. A elevação do estriol sérico é freqüentemente vista em mulheres com LES, e algumas vezes está associada à atividade da doença ${ }^{(26)}$. A ação estrogênica excessiva em pacientes com LES tem um papel essencial na produção da IL-10 ${ }^{(27)}$. Alguns estudos sugerem que altas doses de estrogênio exógeno podem agravar o $\mathrm{LES}^{(26)}$. Mas existem estudos controversos que mostram mulheres portadoras de LES com níveis sorológicos significativamente menores de estradiol e progesterona que os controles, tendo o aumento dos níveis de estrogênio e progesterona ocorrido no terceiro trimestre, ocasião em que as pacientes com LES apresentaram os mais baixos níveis de imunoglobulina no soro e de atividade da doença. Esses dados desafiam os estudos que apóiam a direta relação entre o aumento dos níveis dos hormônios esteroidais e a atividade do Lúpus e ensejam o questionamento sobre a capacidade de o estrogênio e a progesterona suprimirem ou não a atividade da doença ${ }^{(28)}$.

A gravidez resulta em uma supressão da resposta imune celular, mas também na preservação e algumas vezes aumento da imunidade humoral. Estrogênio e progesterona têm efeitos diferentes na regulação do equilíbrio Th1/Th2. Progesterona aumenta a produção de IL-4 e IL-10 pelas células $\mathrm{T}$, promovendo assim a diferenciação das células T. Um estudo mostrou que pacientes com LES responderam ao tratamento com estrogênio com uma redução da produção de TNF pelas células do sangue. TNF é um dos fatores de regulação da apoptose, a falha para manter essa produção durante a gestação pode perturbar a atividade celular imunocompetente da apoptose no LES e assim aumentar a autoimunidade. Alguns investigadores acreditam que as mudanças induzidas pela gestação na resposta Th2 é um fator contribuinte para a atividade observada em pacientes gestantes com $\operatorname{LES}^{(26)}$.

Estrogênio estimula a secreção de prolactina. Prolactina é um hormônio polipeptídico secretado pela glândula pituitária anterior, que aumenta durante a gravidez e no período da lactação ${ }^{(26)}$. Tem efeito sobre as células B e células $\mathrm{T}^{(29)}$, está envolvida na proliferação e diferenciação celular, e tem múltiplos efeitos sobre o sistema imune. Prolactina estimula a resposta imune. Diversos investigadores verificaram níveis elevados de prolactina em pacientes com LES em ambos os sexos. Alguns estudos associaram a elevação dos níveis de prolactina com índices clínicos e laboratoriais de atividade da doença. Foi sugerido que a prolactina está envolvida na atividade do LES, observada na gravidez ${ }^{(26,29)}$.

As mudanças hormonais da gravidez podem agravar os sintomas do LES. Os efeitos induzidos pela gestação com 
aumento das citocinas Th2, tal como IL-4 e IL-10, podem desencadear as manifestações do LES que são dependentes da resposta imune humoral ${ }^{(26)}$.

Dados recentes mostram diminuição significativa da concentração de hormônios esteroidais no soro das pacientes com LES no terceiro trimestre de gestação, fator que poderia ser responsável pela surpreendente diminuição de recaídas observada por alguns autores neste período de gestação nas pacientes com LES ${ }^{(30)}$.

A imunidade na gestação com LES precisa ser mais bem estudada; talvez o melhor controle da resposta imune seja a solução para se evitar que algumas pacientes com LES entrem em atividade da doença durante a gestação.

Função autoimune anormal tem sido associada com falhas no processo de reprodução há longa data. De fato, muitas das condições médicas historicamente associadas com abortamentos (e alguns casos de infertilidade) são atualmente reconhecidas como relacionadas a uma etiologia autoimune $^{(31)}$.

Estudos recentemente publicados têm demonstrado o papel do leito placentário na patogênese de diversas desordens da gestação. Consideráveis avanços têm sido alcançados com o melhor entendimento das complexas relações entre o trofoblasto fetal e materno, assim como do leito placentário durante a gestação normal. Diversas afecções da gestação, incluindo a pré-eclâmpsia e o crescimento intrauterino restrito, parecem ser conseqüência direta da pouca afinidade entre o sistema celular materno-fetal dentro do leito placentário. A síndrome materna caracterizada pela pré-eclâmpsia aparentemente é mediada pela isquemia placentária secundária à insuficiência das artérias espirais. Tal processo pode ser conseqüente a fatores que, ou limitam o tamanho das artérias espirais (placentação insuficiente), ou obstruem as mesmas. As manifestações clínicas da síndrome pré-eclâmptica ocorrem posteriormente, como

\section{REFERÊNCIAS}

1. Medawar PB: Some immunological and endocrinological problems raised by the evolution of viviparity in vertebrates. Symp Soc Exp Biol 44: 320-38, 1953.

2. Zhou Y, Fisher SJ, Janatpour M, et al: Human cytotrophoblasts adopt a vascular phenotype as they differentiate. A strategy for successful endovascular invasion? J Clin Invest 99: 2139-51, 1997.

3. Cross JC, Werb Z, Fisher SJ: Implantation and the placenta: key pieces of the development puzzle. Science 266: 1508-81, 1994.

4. Sacks G, Sargent I, Redman C: An innate view of human pregnancy. Immunol Today 20: 114-8, 1999.

5. Moffett-King A: Natural killer and pregnancy. Nat Rev Immunol 2: 656-63, 2002 . conseqüência da isquemia placentária por disfunção difusa do endotélio materno ${ }^{(32)}$.

O conceito de isquemia placentária tem recebido ampla aceitação entre as diversas teorias existentes e colocando-se à frente para explicar a síndrome. O estabelecimento da pré-eclâmpsia parece ocorrer em dois estágios: (1) uma isquemia placentária absoluta ou relativa por alteração vascular ou placenta hipertrófica, ou como parece ser mais freqüente, secundária a defeito de implantação, particularmente por causas intrínsecas a invasão trofoblástica; (2) doença endotelial difusa. A conexão entre esses dois estágios não foi ainda totalmente esclarecida. O sistema imune materno, que é sobrecarregado durante todos os estágios da gestação normal, está diretamente implicado no desencadeamento do quadro. Os antígenos fetoplacentários podem ser pouco reconhecidos. Esse defeito de reconhecimento poderia levar às anormalidades da invasão trofoblástica observadas na pré-eclâmpsia. Alguns tipos de pré-eclâmpsia podem ser de natureza auto-imune, com a produção de anticorpos direcionados contra certos tipos de fosfolipídios ou constituintes trofoblásticos. Um desequilíbrio entre mecanismos de oxidação e antioxidação envolvendo neutrófilos pode levar à agressão do endotélio observada na pré-eclâmpsia. A pré-eclâmpsia pode representar uma forma de imunedistrofia, com a extensiva produção de citoquinas locais, direcionadas contra o trofoblasto. Assim parece que, durante a gestação, o sistema imune desenvolve mecanismos de proteção que, quando oprimido ou inadequado, permite que ocorra a pré-eclâmpsia. Em outros casos, o mesmo sistema pode fazer parte da cascata de agressões que culminam com as alterações encontradas. A integração dessas anormalidades em modelos fisiopatológicos, pode ser de auxílio na melhoria da classificação desses distúrbios, potencialmente vindo a permitir um manuseio preventivo e terapêutico mais adequado da pré-eclâmpsia ${ }^{(33)}$.

6. Henderson TA, Saunders PTK, Moffett-King A, Groome NP, Critchley HOD: Steroid receptor expression in uterine natural killer cells. J Clin Endocrinol Metab 88: 440-9, 2003.

7. Lobo SC, Huang STJ, Germeyer A, et al: The immune environment in human endometrium during the window of implantation. Am J Reprod Immunol 52: 244-51, 2004.

8. Kitaya K, Yasuda J, Yagi I, Tada Y, Fushiki S, Honjo H: IL-15 expression at human endometrium and decidua. Biol Reprod 63: 683-7, 2000.

9. Drake PM, Gunn MD, Charo IF, et al: Human placental cytotrophoblasts attract monocytes and CD56 ${ }^{\text {bright }}$ natural killer cells via the actions of monocyte inflammatory protein $1 \alpha$. J Exp Med 193: 1199-212, 2001. 
10. Koopman LA, Kopcow HD, Rybalov B, et al: Human decidual natural killer cells are a unique NK cell subset with immunomodulatory potential. J Exp Med 198: 1201-12, 2003.

11. Billington WD: The normal fetomaternal immune relationship. Baillieres Clin Obstet Gynaecol 6: 417-38, 1992.

12. Verma S, Hiby SE, LokeYW, King A: Human decidual natural killer cells express the receptor for and respond to the cytokine interleukin 15. Biol Reprod 62: 959-68, 2000.

13. King AE, Critchley HOD, Kelly RW: Innate immune defences in the human endometrium. Reprod Biol Endocrinol 1: 116, 2003.

14. Sigal LH: Basic science for the clinician 33 - interleukins of current clinical relevance (Part I). J Clin Rheumatol 10: 353-9, 2004.

15. Zenclussen AC, Fest S, Busse P, Joachim R, Klapp BF, Arck PC: Questioning the Th1/Th2 paradigm in reproduction: Peripheral levels of IL-12 are down-regulated in miscarriage patients. Am J Reprod Immunol 48: 245-51, 2002.

16. Parr EL, Chen HL, Parr MB, Hunt JS: Synthesis and granular localization of tumor necrosis factor-alpha in activated NK cells in the pregnant mouse uterus. J Reprod Immunol 28: 31-40, 1995.

17. Zicari A, Ticconi C, Realacci $\mathrm{M}$, et al: Hormonal regulation of cytokine release by human fetal membranes at term gestation: effects of oxytocin, hydrocortisone and progesterone on tumour necrosis factor- $\alpha$ and transforming growth factor- $\beta 1$ output. J Reprod Immunol 56: 123-6, 2002.

18. Sacks GP, Redman CWG, Sargent IL: Monocytes are primed to produce the Th1 type cytokine IL-12 in normal human pregnancy: an intracellular flow cytometric analysis of peripheral blood mononuclear cells. Clin Exp Immunol 131: 490-7, 2003.

19. Peltier MR: Immunology of term and labor. Reprod Biol Endocrinol 1: $122,2003$.

20. Cutolo M, Sulli A, Villaggio B, Seriolo B, Accardo S: Relations between steroid hormones and cytokines in rheumatoid arthritis and systemic lupus erythematosus. Ann Rheum Dis 57: 573-7, 1998.

21. Marzi M, Vigano A, Trabattoni D, et al: Characterization of type 1 and type 2 cytokine production profile in physiologic and pathologic human pregnancy. Clin Exp Immunol 106: 127-33, 1996.
22. Cadet P, Rady PL, Tyring SK, Yandell RB, Hughes TK: Interleukin10 messenger ribonucleic acid in human placenta: Implications of a role for interleukin-10 in fetal allograft protection. Am J Obstet Gynecol 173: 25-9, 1995.

23. Elenkov IJ, Wilder RL, Bakalov VK, et al: IL-12, TNF- $\alpha$, and hormonal changes during late pregnancy and early postpartum: Implications for autoimmune disease activity during these times. J Clin Endocrinol Metab 86: 4933-8, 2001.

24. Baxter AG, Smyth MJ: The role of NK cells in autoimmune disease. Autoimmunity 35: 1-14, 2002.

25. Szyper-Kravitz M, Zandman-Goddard G, Lahita RG, Shoenfeld Y: The neuroendocrine-immune interactions in systemic lupus erythematosus: A basis for understanding disease pathogenesis and complexity. Rheum Dis Clin N Am 31: 161-75, 2005.

26. Ostensen M: Sex hormones and pregnancy in rheumatoid arthritis and systemic lupus erythematosus. Ann NY Acad Sci 22; 876: 131-43, 1999

27. Draca S: Is pregnancy a model how we should control some autoimmune diseases? Autoimmunity 35: 307-12, 2002.

28. Warren JB, Silver RM: Autoimmune disease in pregnancy: systemic lupus erythematosus and antiphospholipid syndrome. Obstet Gynecol Clin N Am 31: 345-72, 2004.

29. Leaños-Miranda A, Pascoe-Lira D, Chávez-Rueda KA, BlancoFavela F: Persistence of macroprolactinemia due to antiprolactin autoantibody before, during, and after pregnancy in a woman with systemic lupus erythematosus. J Clin Endocrinol Metab 86: 2619-24, 2001.

30. Doria A, Ghirardello A, Iaccarino L, et al: Pregnancy, cytokines, and disease activity in systemic lupus erythematosus. Arthritis Rheum 51: 989-95, 2004.

31. Gleicher N: Autoantibodies in normal and abnormal pregnancy. Am J Reprod Immunol 28: 269-73, 1992.

32. Redman CW: Immunological aspects of pre-eclampsia. Baillieres Clin Obstet Gynaecol 6: 601-15, 1992.

33. Vinatier D, Monnier JC, Pre-eclampsia: physiology and immunological aspects. Eur J Obest Reprod Biol 61: 85-97, 1995. 